\title{
Estudio preliminar de los métodos de evaluación del control compartido
}

\author{
Joseba Sarabia, Mauricio Marcano, Joshué Perez Rastelli, Sergio Diaz Briceño, Asier Zubizarreta \\ TECNALIA, Basque Research and Technology Alliance (BRTA) \\ Escuela de Ingeniería de Bilbao (EIB) \\ joseba.sarabia@tecnalia.com, mauricio.marcano@tecnalia.com, joshue.perez@tecnalia.com, \\ sergio.diaz@tecnalia.com, asier.zubizarreta@ehu.eus
}

\section{Resumen}

La conducción automatizada es un área en auge, con múltiples ventajas para el transporte de personas y mercancías. A pesar de que el objetivo es lograr una automatización total, los niveles intermedios de automatización requieren desarrollar el concepto de Control Compartido, en el que el vehículo y el conductor ejercen simultáneamente una acción de control sobre uno (o más) de los actuadores del vehículo (ej., el volante). Con el fin de poder definir metodologías adecuadas para evaluar y verificar estos sistemas, en este trabajo se analizan las métricas más relevantes de la literatura y se propone una metodología que constituirá la base para futuros desarrollos.

Palabras clave: Conducción automatizada, Control Compartido, Métricas de Evaluación

\section{Introducción}

La conducción automatizada (Automated Driving en inglés, $\mathrm{AD}$ ), ha captado una gran atención en la actualidad. Los principales fabricantes de automoción ya están testeando sus prototipos y ciertos sistemas de ayudas a la conducción (ADAS). La oferta de estos es cada vez más amplia. Ya no sólo se trata de sistemas ABS, sistemas de estabilidad ESP o el control de crucero. Actualmente, existen modelos comerciales con sistemas de ayuda activa a la conducción como los sistemas de mantenimiento de carril (LKA) o el aparcamiento automatizado.

$\mathrm{Si}$ bien estos sistemas traen consigo beneficios como una mejora en la fluidez del tráfico [10], una reducción en el consumo de combustible [3] o la reducción de la densidad de tráfico en las ciudades y el uso compartido de vehículos [12], el principal beneficio que aportan está en la seguridad. Los coches con sistemas de ayuda a la conducción son (y serán $[4,5,7]$ ) mucho más seguros que los únicamente controlados por personas. Numerosos estudios demuestran que un alto porcentaje de los accidentes de carretera están causados por errores humanos $[9,8]$. Ya sean distracciones, conducir en un estado inadecuado o hacer otras tareas paralelamente a la conducción son causas que comprenden más del $90 \%$ de los siniestros [29, 24].

La mejor forma de entender la conducción automatizada es a través de los niveles definidos por la Sociedad de Ingenieros de Automoción (SAE). En 2014 la SAE estableció una terminología común que fue acogida por tanto gobiernos como la industria [1]. Esta especifica 6 niveles de automatización, desde "sin automatizar" (nivel 0) hasta "automatización completa" (nivel 5). Los niveles del 1 al 3 aumentan progresivamente el uso de la automatización, pero implican al conductor en la tarea de conducción. En el nivel 3 los vehículos deben ser capaces de conducir solos en determinadas situaciones, siempre y cuando haya un conductor que se haga cargo en situaciones de emergencia. Algunas compañías como Tesla y GM lanzaron en su momento los primeros modelos de nivel 3, pero por problemas legales en accidentes tomaron la precaución de llamarlo nivel 2 [13], donde el responsable último es el conductor y no el vehículo. En la actualidad, muchas compañías abogan por saltarse el nivel 3, y desarrollar directamente los niveles 4 y 5 , donde el vehículo es completamente responsable de la tarea de conducción [18]. El nivel 4 se ha visto retrasado a una fase donde requiere un mayor estudio y desarrollo.

Por otra parte, estos niveles no consideran una interacción continua y constante entre la automatización y el conductor. En este sentido, la principal rama de $\mathrm{AD}$ que estudia la interacción entre el conductor y el sistema es el Control Compartido (Shared Control, SC) [15, 17]. Este concepto recoge que tanto el conductor como el vehículo deben controlar simultáneamente el volante o los pedales, lo que es un requisito en etapas intermedias de automatización. Esta cooperación requiere un entendimiento de la intención de ambas partes, dado que ambas están capacitadas para tomar decisiones sobre el control del vehículo. Como se muestra en la (Figura 1), los principales agentes son el sistema automatizado y el conductor. Dentro del marco del SC, ambos cuentan con las herramientas necesarias para guiar el vehículo.

El vehículo automatizado, por un lado, cuenta con 
el esquema convencional de 6 bloques (incluyendo el módulo de actuación) [16] donde el vehículo recibe la información del entorno, genera una trayectoria acorde y la sigue mediante el lazo de control. El conductor, por el otro, está sujeto a la monitorización de su estado a través del Sistema de Monitorización del conductor (o Driver Monitoring System, DMS). Además, el sistema de interacción humano-máquina (o Human Machine Interface, HMI) le permite intercambiar información con el vehículo de forma bidireccional. Entre ambos agentes se encuentra el módulo de arbitraje. Mediante este se regula la cooperación entre ambos sistemas. En función del estado y la actitud del conductor, se adecua el nivel de asistencia ofrecida por el vehículo, de manera que se cumplan los objetivos de conducción previamente definidos en el diseño del sistema.



Figura 1: Marco del Control Compartido.

Diferentes sistemas basados en SC han sido desarrollados en los últimos años con múltiples aplicaciones para vehículos automatizados. Sin embargo, la mayoría han sido probados en plataformas de simulación driver-in-the-loop (DiL). Por lo tanto, el siguiente avance tecnológico consiste en una futura implementación en vehículos reales. Para ello, un paso fundamental es establecer una metodología de evaluación y validación bien definida de dichas funcionalidades.

En el mundo de automoción, la validación de componentes sigue una estructura muy específica y estandarizada: El método $\mathrm{V}$ de verificación y validación. Esto es un proceso secuencial marcadamente rígido que describe las actividades y resultados que deben producirse en el desarrollo de componentes de automoción. Se trata de un proceso lento, pero con resultados contrastados. El desarrollo de ADAS, en cambio, sigue una estructura diferente. Al tratarse de una tecnología no madura, cada año existen nuevas variantes de cada sistema, por lo que deben desarrollarse nuevas formas de validarlos. Tanto es así que instituciones internacionales como la SAE, ISO, UNE y NHTSA están lanzando y actualizando frecuentemente los nuevos estándares de los ADAS [2, 1, 11], y han hecho un llamamiento a las instituciones relacionadas para participar en su desarrollo.

Dentro de este contexto, el SC es un caso complejo, ya que requiere de la validación de los sistemas con el conductor dentro del loop (DiL) de control. Esto implica que asegurar el funcionamiento del sistema no es suficiente, sino que debe ser a su vez capaz de ajustarse a las decisiones y necesidades de cualquier conductor. Numerosos estudios apuntan a hacer modificaciones en la estructura de validación de estas tecnologías, adoptando estrategias "Human-Centred Design" (HCD) [19]. De esta manera el modo de validación se ajusta más a las condiciones del usuario. HCD concretamente es una metodología que implica al usuario final en todo el proceso de desarrollo, desde la gestación de ideas iniciales y requisitos, hasta la validación. Utiliza una estructura iterativa, donde cada sistema se actualiza en base a las valoraciones de sus usuarios. La validación se lleva a cabo mediante tres métodos: Testeo con usuarios, inspecciones basadas en pautas de uso y accesibilidad, y monitorización en uso prolongado. Actualmente, HCD es una metodología inmadura, en algunos casos acuñada más bien como una filosofía, de uso mayoritario en investigación. Es por esto que su utilidad reside en marcar las pautas de lo que se posteriormente se definirá como la metodología de validación del SC.

Tomando el HCD y el llamamiento de la SAE como base, este documento muestra una propuesta de metodología de validación para los sistemas de SC basada en 5 pasos, definiendo métricas y procedimientos para sentar una base futura a los desarrollos en el área.

El resto del artículo está estructurado de la siguiente forma: En la Sección 2, se detalla la estructura general de la metodología, que abarca 5 fases, detalladas en las secciones 3-6. La sección 7 resume las ideas más importantes.

\section{Visión General de la Metodología}

La metodología propuesta, basada en el llamamiento de la SAE [6] se muestra en la Figura 2. Está compuesta por 5 bloques, con una estructura secuencial iterativa. En primer lugar, se describen los objetivos que el sistema busca alcanzar. En segundo lugar, se definen las métricas para alcanzar dichos objetivos. Esto se trata de un proceso iterativo, por lo que en la primera iteración se definen unas métricas basadas en la experiencia. En el siguiente paso, se describe el entorno operativo (Operational Design Domain en inglés, ODD) 
en el que se deben cumplir estos objetivos. Posteriormente, se hace un análisis de las métricas obtenidas y, en función del resultado, se modifican o añaden nuevas métricas. Se repite este proceso hasta obtener unas métricas que ofrezcan unos resultados satisfactorios. Estas fases serán analizadas en las secciones siguientes.

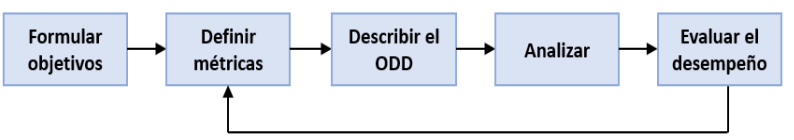

Figura 2: Proceso recomendado por la SAE para la obtención de pruebas de desempeño [6].

\section{Formulación de Objetivos}

El primer paso es definir los beneficios que el SC debe reportar. Para ello, se han clasificado en cuatro grupos los principales objetivos estudiados en el estado del arte del SC. Si bien pudiera existir un quinto que es el relacionado con los aspectos legales y la gestión de la responsabilidad, ese apartado queda fuera de este estudio:

- Seguridad: Es el objetivo principal para cualquier sistema en automoción, y el más difícil de cumplir en una arquitectura con dos agentes tomando decisiones al mismo tiempo. En situaciones de emergencia se debe establecer una gestión de la autoridad clara sobre el control del vehículo, siendo el conductor el principal capacitado para asumir el control, y el vehículo, en caso de que el conductor muestre signos de no estar capacitado para ello.

- Control: El sistema debe estar capacitado para mantener el control del vehículo siempre que esté activo. Ya sea en situaciones de conducción normal como en situaciones donde el conductor pudiere perder el control, el sistema protegería al conductor. El hecho de que el sistema pueda corregir al conductor en determinadas situaciones requiere que este deposite su confianza en el sistema [28]. Darle confianza al usuario implica dos cosas. En primer lugar, el sistema debe mostrar fiabilidad y fallar el mínimo número de veces posible. En segundo lugar, el sistema debe mostrar un desempeño confiable. Es decir, que el usuario entienda en todo momento las acciones del sistema y sienta que estas son correctas.

- Cooperación: El conductor debe ser capaz de interpretar en todo momento las decisiones del vehículo de forma intuitiva. Para facilitar esa labor, el intercambio de información entre ambos es primordial. Esta función la cumple el HMI, que gana un especial protagonismo en el SC [27].

- Aceptación: Al tratarse de un sistema orientado al conductor, es importante que este genere una sensación de satisfacción por parte del usuario, algo que haga que prefiera usar dicho sistema a no usarlo. Este objetivo está compuesto de una serie de subobjetivos como el confort del conductor, el esfuerzo o carga de trabajo que requiera utilizar el sistema, la sensación de seguridad que tenga al utilizarlo (que no necesariamente está asociada con la seguridad real) e incluso el nivel de entretenimiento que el sistema pueda ofrecer [28].

\section{Definición de Métricas}

Según el estado del arte, las formas más comunes de clasificar los diferentes tipos de métricas mediante dos enfoques. Por un lado, se pueden clasificar en función de la realidad y la percepción de esta. Es decir, en cuanto a si se trata de una métrica objetiva o subjetiva. En el primer caso se incluyen lo relativo a las medidas obtenidas de forma cuantitativa. Aquí se encuentran las variables relacionadas con el vehículo y las obtenidas por el DMS, del conductor. En cuanto a las variables subjetivas, se tratan de las relacionadas con la percepción del conductor. La mayoría de los estudios del SC incluyen una serie de cuestionarios para obtener esta información. Por el otro lado, se pueden clasificar en función del objeto de estudio, como se muestra en la Tabla 1. En este caso, se trata del vehículo, el conductor y la situación que los rodea. A continuación, se detallan las más relevantes.

\subsection{Métricas del vehículo}

En cuanto al vehículo, el desempeño de la conducción es el concepto más estudiado. Este hace referencia a la precisión con la que el vehículo es capaz de llevar a cabo la tarea deseada. Esta métrica está estrechamente relacionada con la conducción automatizada. Principalmente, trata de medir la eficiencia en el seguimiento de carril. En relación al SC, se encuentran métricas relacionadas con la estrategia colaborativo, tales como el nivel de asistencia proporcionado por el vehículo, el fuerza aplicado por el sistema en el actuador y la frecuencia de intervenciones de este. Finalmente, una visión subjetiva de todas ellas reflejan el confianza en el sistema que el vehículo es capaz de ofrecer [20, 21, 22, 23]. 
Cuadro 1: Tipos de métricas según el agente de estudio.

\begin{tabular}{|c|c|c|}
\hline Vehículo & Conductor & Entorno \\
\hline Desempeño de la conducción & Guiado del vehículo & Evaluación de la situación \\
Seguimiento de carril & Capacidad del conductor & Probabilidad de accidente \\
Transferencia de control & Modificadores de la capacidad del conductor & Grado de exigencia \\
Estrategia colaborativo & Estado del conductor & Dificultad \\
Nivel de asistencia & Carga de trabajo & Criticidad \\
Confianza en el sistema & Demanda visual & Evasión de obstáculos \\
Frecuencia de intervenciones del sistema & Nivel de atención & Maniobras \\
Fuerza del sistema en el actuador & Respuesta ante sucesos & Tareas secundarias \\
\hline
\end{tabular}

\subsection{Métricas del conductor}

En lo relativo a las métricas del conductor, la actividad de la conducción se clasifica en dos modos. Por un lado, está el guiado del vehículo, donde se evalúa la capacidad del conductor para manejar el vehículo, ya sea en condiciones normales, sin alteraciones por parte del entorno ni del propio conductor, o en condiciones donde sí se modifique la capacidad del conductor. Aquí entran métricas relacionadas con el estado del conductor (somnolencia, fatiga, estrés, cansancio, etc.) [29], su carga de trabajo [25] y la demanda visual requerida [27]. Por otro lado, están los eventos concretos (como podrían ser una transición de manual a automático, realizar alguna tarea secundaria, evitar un accidente, etc.). En este caso se miden variables como el nivel de atención del conductor, la respuesta ante sucesos y el tiempo de reacción.

\subsection{Métricas del entorno}

Finalmente, respecto a las métricas del entorno, se pretende hacer una evaluación de la situación, donde se valore el grado de exigencia que esta requiere, su nivel de dificultad, y si se trata de una situación crítica o no. También se busca medir probabilidad de accidente de una situación [30], que principalmente suelen estar causados por distracciones, las cuales a su vez son la consecuencia del conductor realizando tareas secundarias.

\section{Descripción del Entorno: Fuentes de información}

Una vez obtenidas dichas métricas, es necesario establecer el entorno en el que desean ser estudiadas. Ya sea una situación de carretera como la autopista, un caso de uso de un conductor distraído o la gestión de una maniobra como un cambio de carril, en cualquier caso, existen métodos para la obtención de información de dichas situaciones [31].

En primer lugar, están los estudios en carretera. Consisten en utilizar un vehículo equipado por la institución investigadora para llevar a cabo el experimento. Se trata de los estudios más complejos de realizar, ya que requieren de los permisos legales para llevar un vehículo experimental a la carretera. Por ello, deben estar equipados con sistemas de seguridad y personas cualificadas para dichas labores. Los principales casos de uso de estas pruebas consisten en pruebas de baja velocidad, donde se prueban métricas orientadas al vehículo. Esto se debe a que las pruebas orientadas al conductor pueden requerir que este pierda el control (o simule hacerlo) durante la conducción.

En segundo lugar, se encuentran los estudios naturalistas [32]. Estos son los más similares a los anteriores, en tanto que también se llevan a cabo en vehículos reales. Se trata de colocar sistemas de medición en los vehículos de los particulares dispuestos a hacer las pruebas. Esto permite que se hagan estudios más amplios, con muchos más kilómetros recorridos. El principal inconveniente es la distribución de los equipos en estos vehículos, y la obtención de datos estandarizada. Un ejemplo muy común de estos son los estudios del conductor, donde se le coloca una cámara para monitorizar su actividad. En este caso en particular, el inconveniente principal suele ser la ubicación de la cámara. Ya que posteriormente todos los datos obtenidos tendrán el mismo post-procesado, y pequeños cambios en la posición u orientación puede derivar en errores de precisión en las medidas de sistemas de IA [33].

En tercer lugar, están los estudios más frecuentes, que son los llevados a cabo en un simulador. Estos permiten la máxima flexibilidad a la hora de diseñar las situaciones y añadir eventos, dado que siempre se trabaja sobre un entorno seguro. Es por esto que la mayor parte de los estudios de control compartido se llevan a cabo en simuladores [34, 35, 36]. Abarca estudios de todo tipo, ya sea de métricas del vehículo, del conductor y del entorno. A pesar de ello, los resultados corren el riesgo de estar sesgados, ya que no se realizan en condiciones reales para ninguno de los tres tipos 
de métricas.

En cuarto lugar, están los estudios de las bases de datos de las instituciones. Gobiernos como el de EEUU ponen a disposición de las instituciones los datos de accidentes de carretera para su estudio. Esto permite obtener información de casos reales de situaciones de emergencia, probablemente los casos más difíciles de estudiar en pruebas en carretera o en simuladores. De aquí también es posible extraer información de los tres tipos de métricas, ya sea porque el accidente se haya dado por un fallo del vehículo, del conductor y de la situación que se diese. De todos modos, pueden llegar a ser más difíciles de estudiar dado que no tienen por qué adecuarse exactamente al caso de estudio concreto, por lo que las conclusiones obtenidas pueden ser sesgadas.

Finalmente, se encuentran los estudios llevados a cabo mediante encuestas. Estos permiten obtener la perspectiva subjetiva de los conductores. Pueden llegar a ser muy útiles para medir el nivel de confianza y de aceptación de un sistema, y también para relacionar esa perspectiva subjetiva con datos empíricos obtenidos en métricas objetivas. Ofrecen un amplio abanico de posibilidades, pero también requieren de un uso extensivo. Es decir, es necesario que la muestra poblacional encuestada sea lo suficientemente extensa como para que los datos obtenidos sean asimilables al resto de la población. También es habitual que se tomen datos personales de los participantes (como edad, años de experiencia al volante, sexo, capacidades sensoriales, estado de salud, etc.) para poder crear perfiles de conducción.

\section{Análisis y Evaluación del desempeño}

En muchos casos, el análisis individual de las métricas ofrece una visión sesgada. Es cuando estas se interrelacionan cuando realmente se consigue inferir información. Como se mencionaba previamente, resulta muy útil relacionar estudios empíricos con resultados de encuestas, ya que amplían la perspectiva y las conclusiones obtenidas. En cuanto a las pruebas empíricas, ya sea en estudios en carretera o en simulador, es muy importante acotar el alcance y las condiciones de la prueba, de forma que esta quede segmentada y sea fácilmente replicable por un estudio de otra institución. Respecto a los cuestionarios, resulta conveniente tomar tantos datos como resulte conveniente de los participantes, para definir en la mejor medida los perfiles de cada conductor.

En cuanto el tamaño de la muestra poblacional, en términos de estadística se estima que alrededor de
200 participantes [37] es un número adecuado para poder inferir información de toda la población, y a partir de 2000 el margen de error se estima como nulo. Estas cifras son viables a la hora de realizar cuestionarios. A la hora de realizar pruebas en carretera o simulador, en cambio, puede resultar problemático montar a tantos participantes en el asiento del conductor. Por ello, es habitual que se hagan estudios más reducidos (entre 15 y 50 participantes), donde los perfiles estudiados sean específicos respecto a lo que se busque medir.

A la hora de comparar los datos obtenidos de diferentes conductores, es frecuente es uso de estudios ANOVA (Análisis de la Varianza) [38] para probar la hipótesis de si las medias de una o varias poblaciones son iguales o no.

\section{Conclusiones}

La conducción automatizada actualmente cuenta con varios años de desarrollo y está entrando en una fase de evaluación de sus sistemas. Comparativamente, el control compartido es una disciplina compleja de estudio y que, como se muestra en este artículo, toma de una serie de consideraciones diferentes a la del vehículo automatizado. Por parte del vehículo, requiere de un sistema que sea capaz de transferir el control del conductor al sistema de forma fluida. Por parte del conductor, se realiza un estudio más exhaustivo de sus capacidades, dando pie a que en un futuro se alcance un consenso de qué requisitos se le pueden exigir a una persona a la hora de gestionar la responsabilidad de la tarea de conducción. Como propuesta para trabajos futuros, se pretende ampliar el estudio de las métricas del control compartido, de manera que se definan las principales variables medibles y se relacionen con los métricas y los objetivos aquí mostrados, de manera que se obtenga un extenso estudio del estado del arte del diseño y evaluación de los sistemas de control compartido. Además, también es ampliable desde el punto de vista legal, donde todavía no se ha definido una forma clara de definir los tiempos de los que dispone el conductor para tomar el control en situaciones donde el vehículo ya no sea capaz de tomar dicha responsabilidad.

\section{Agradecimientos}

Este trabajo cuenta con el apoyo del proyecto HADRIAN, el cual ha recibido financiación del programa de investigación e innovación Horizonte 2020 de la Unión Europea en virtud del acuerdo de subvención No 875597.

\section{English summary}




\section{Preliminary study of the evaluation methods for Shared Control}

\begin{abstract}
Focus on Automated Driving has increased in the last years due to the advantages in people and good transports. Although full automatization is the goal of vehicle manufacturer's, the technological development requires to share control between the vehicle and the driver in intermediate automation levels. In order to define proper validation methodologies for this complex strategy, in this paper the most relevant works are analysed and a methodology is proposed to set the basis for future developments.
\end{abstract}

Keywords: Automated Driving, Shared Control, evaluation metrics.

\section{Referencias}

[1] "J3016C: Taxonomy and Definitions for Terms Related to Driving Automation Systems for On-Road Motor Vehicles - SAE International," 2021. Accessed: May 24, 2021. [Online]. Available: https://www.sae.org/ standards/content/j3016202104/

[2] "Automated Vehicles — National Highway Traffic Safety Administration (NHTSA)." ht tps://one.nhtsa.gov/Research/Crash+A voidance/Automated+Vehicles (accessed Aug. 31, 2020).

[3] M. S. Young, S. A. Birrell, and N. A. Stanton, "Safe driving in a green world: A review of driver performance benchmarks and technologies to support 'smart' driving," Appl. Ergon., vol. 42, no. 4, pp. 533-539, May 2011, doi: 10.1016/j.apergo.2010.08.012.

[4] N. A. Stanton and P. Marsden, "From fly-bywire to drive-by-wire: Safety implications of automation in vehicles," Saf. Sci., vol. 24, no. 1, pp. 35-49, Oct. 1996, doi: 10.1016/S09257535(96)00067-7.

[5] N. Highway Traffic Safety Administration and U. Department of Transportation, "TRAFFIC SAFETY FACTS Crash • Stats Critical Reasons for Crashes Investigated in the National Motor Vehicle Crash Causation
Survey," 2015. Accessed: May 24, 2021. [Online]. Available: https://crashstats.nht sa.dot.gov/Api/Public/ViewPublicatio $\mathrm{n} / 81211$

[6] AVSC Best Practice for Metrics and Methods for Assessing Safety Performance of Automated Driving Systems (ADS). AVSC00006202103 2021-03-25. Accessed: May 19, 2021. [Online]. Available: https://www . sae.org/standards/conten t/avsc00006202103/

[7] I. Y. Noy, D. Shinar, and W. J. Horrey, "Automated driving: Safety blind spots," Safety Science, vol. 102. Elsevier B.V., pp. 68-78, Feb. 01, 2018, doi: 10.1016/j.ssci.2017.07.018.

[8] S. Shen and D. M. Neyens, "Assessing drivers' response during automated driver support system failures with non-driving tasks," J. Safety Res., vol. 61, pp. 149-155, Jun. 2017, doi: 10.1016/j.jsr.2017.02.009.

[9] F. Hartwich, M. Beggiato, and J. F. Krems, "Driving comfort, enjoyment and acceptance of automated driving-effects of drivers' age and driving style familiarity," Ergonomics, vol. 61, no. 8, pp. 1017-1032, Aug. 2018, doi: 10.1080/00140139.2018.1441448.

[10] Liu, Peng, Yang, Run, Xu, Zhigang (2018). Public Acceptance of Fully Automated Driving: Effects of Social Trust and Risk/Benefit Perceptions. Risk Analysis, 39(2) (pp. 326-341), ISSN 0272-4332, Wiley, doi:10.1111/risa. 13143

[11] Intelligent transport systems - Low-speed automated driving (LSAD) systems for predefined routes — Performance requirements, system requirements and performance test procedures, Working Document, ISO 22737

[12] S. Shaheen, "Shared Mobility: The Potential of Ridehailing and Pooling," in Three Revolutions, Island Press/Center for Resource Economics, 2018, pp. 55-76.

[13] "Tesla driver dies in first fatal crash while using autopilot mode - Tesla - The Guardian." https : //www. theguardian.com/te chnology/2016/jun/30/tesla-autopil ot-death-self-driving-car-elon-musk (accessed May 31, 2021).

[14] V. A. Banks, A. Eriksson, J. O'Donoghue, and N. A. Stanton, "Is partially automated driving a bad idea? Observations from an on-road study," Appl. Ergon., vol. 68, pp. 138-145, Apr. 2018, doi: 10.1016/j.apergo.2017.11.010. 
[15] D. A. Abbink, M. Mulder, and E. R. Boer, "Haptic shared control: Smoothly shifting control authority?," Cogn. Technol. Work, vol. 14, no. 1, pp. 19-28, Mar. 2012, doi: 10.1007/s10111-011-0192-5.

[16] Vaca-Recalde, Myriam E., Marcano, Mauricio, Sarabia, Joseba, González, Leonardo, Pérez, Joshué, Díaz, Sergio (2020). A FluidHMI Approach for Haptic Steering Shared Control for the HADRIAN Project. Lecture Notes in Computer Science (pp. 417-428), ISSN 0302-9743, Springer International $\mathrm{Pu}-$ blishing, doi:10.1007/978-3-030-50523-3-30

[17] Marcano, Mauricio, Diaz, Sergio, Perez, Joshue, Irigoyen, Eloy (2020). A Review of Shared Control for Automated Vehicles: Theory and Applications. IEEE Transactions on Human-Machine Systems, 50(6) (pp. 475-491), ISSN 2168-2291, Institute of Electrical and Electronics Engineers (IEEE), doi:10.1109/thms.2020.3017748

[18] Z. Lu and J. C. F. de Winter, "A Review and Framework of Control Authority Transitions in Automated Driving," Procedia Manuf., vol. 3, pp. 2510-2517, Jan. 2015, doi: 10.1016/j.promfg.2015.07.513.

[19] Human-centred design processes for interactive systems, Working document, ISO 13407:1999

[20] L. Saleh, P. Chevrel, F. Claveau, J. F. Lafay, and F. Mars, "Shared steering control between a driver and an automation: Stability in the presence of driver behavior uncertainty," IEEE Trans. Intell. Transp. Syst., vol. 14, no. 2, pp. 974-983, 2013, doi: 10.1109/TITS.2013.2248363.

[21] F. Mars, M. Deroo, and J. M. Hoc, "Analysis of human-machine cooperation when driving with different degrees of haptic shared control," IEEE Trans. Haptics, vol. 7, no. 3, pp. 324-333, Jul. 2014, doi: 10.1109/ТOH.2013.2295095.

[22] R. Nishimura, T. Wada, and S. Sugiyama, "Haptic Shared Control in Steering Operation Based on Cooperative Status Between a Driver and a Driver Assistance System," J. Human-Robot Interact., vol. 4, no. 3, p. 19, Mar. 2015, doi: 10.5898/4.3.nishimura.

[23] M. Körber, C. Gold, D. Lechner, and K. Bengler, "The influence of age on the take-over of vehicle control in highly automated driving," Transp. Res. Part F Traffic Psychol. Behav., vol. 39, pp. 19-32, May 2016, doi: 10.1016/j.trf.2016.03.002.
[24] N. Kalra and S. Paddock, Driving to Safety: How Many Miles of Driving Would It Take to Demonstrate Autonomous Vehicle Reliability? RAND Corporation, 2016.

[25] Y. Xiong, J. Gao, Y. Yang, X. Yu, and W. Huang, "Classifying Driving Fatigue Based on Combined Entropy Measure Using EEG Signals," Int. J. Control Autom., vol. 9, no. 3, pp. 329-338, 2016, doi: 10.14257/ijca.2016.9.3.30.

[26] M. Johns et al., "Exploring shared control in automated driving," in ACM/IEEE International Conference on Human-Robot Interaction, Apr. 2016, vol. 2016-April, pp. 91-98, doi: 10.1109/HRI.2016.7451738.

[27] Z. Lu, X. Coster, and J. de Winter, "How much time do drivers need to obtain situation awareness? A laboratory-based study of automated driving," Appl. Ergon., vol. 60, pp. 293-304, Apr. 2017, doi: 10.1016/j.apergo.2016.12.003.

[28] P. Liu, R. Yang, and Z. Xu, "Public Acceptance of Fully Automated Driving: Effects of Social Trust and Risk/Benefit Perceptions," Risk Anal., vol. 39, no. 2, pp. 326-341, Feb. 2019, doi: $10.1111 /$ risa.13143.

[29] V. A. Banks, A. Eriksson, J. O’Donoghue, and N. A. Stanton, "Is partially automated driving a bad idea? Observations from an on-road study," Appl. Ergon., vol. 68, pp. 138-145, Apr. 2018, doi: 10.1016/j.apergo.2017.11.010.

[30] P. Papantoniou, E. Papadimitriou, and G. Yannis, "Review of driving performance parameters critical for distracted driving research," in Transportation Research Procedia, Jan. 2017, vol. 25, pp. 1796-1805, doi: 10.1016/j.trpro.2017.05.148.

[31] N. Das, E. Ohn-Bar, and M. M. Trivedi, "On Performance Evaluation of Driver Hand Detection Algorithms: Challenges, Dataset, and Metrics," in IEEE Conference on Intelligent Transportation Systems, Proceedings, ITSC, Oct. 2015, vol. 2015-Octob, pp. 2953-2958, doi: 10.1109/ITSC.2015.473.

[32] "The 100-Car Naturalistic Driving Study Phase II-Results of the 100-Car Field Experiment," 2006.

[33] P. Knoll, "Driving assistance systems," in Brakes, Brake Control and Driver Assistance Systems, Springer Fachmedien Wiesbaden, 2014, pp. 180-185. 
[34] F. Naujoks, C. Purucker, K. Wiedemann, A. Neukum, S. Wolter, and R. Steiger, "Driving performance at lateral system limits during partially automated driving," Accid. Anal. Prev., vol. 108, pp. 147-162, Nov. 2017, doi: 10.1016/j.aap.2017.08.027.

[35] A. T. Nguyen, C. Sentouh, and J. C. Popieul, "Sensor Reduction for DriverAutomation Shared Steering Control via an Adaptive Authority Allocation Strategy," IEEE/ASME Trans. Mechatronics, vol. 23, no. 1, pp. 5-16, Feb. 2018, doi: 10.1109/TMECH.2017.2698216.

[36] T. Saito, T. Wada, and K. Sonoda, "Control Authority Transfer Method for AutomatedTo-Manual Driving Via a Shared Authority Mode," IEEE Trans. Intell. Veh., vol. 3, no. 2, pp. 198-207, Jun. 2018, doi: 10.1109/TIV.2018.2804167.

[37] "Cuadernos Técnicos Conceptos Básicos de Muestreo," 2014. https://www.icjce.es/a djuntos/AuditoresCuaderno21.pdf

[38] Ronald E. Walpole; Raymond H. Myers; Sharon L. Myers; Linda Margarita Medina Herrera, Ed., Probabilidad y estadística para ingeniería y ciencias, 9th ed. Ciudad de Mexico: Pearson, 2012.

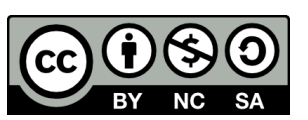

C) 2021 by the authors. Submitted for possible open access publication under the terms and conditions of the Creative Commons Attribution CC BY-NC-SA 4.0 license (https://creativecommons.org/licenses/by-ncsa/4.0/deed.es). 